\title{
Experimental study of the effect of local atomic ordering on the energy band gap of melt grown InGaAsN alloys
}

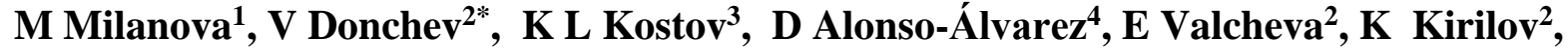 I Asenova², I G Ivanov ${ }^{5}$, S Georgiev ${ }^{2}$ and N Ekins-Daukes ${ }^{4}$}

${ }^{1}$ Central Laboratory of Applied Physics, 59 St. Petersburg blvd, 4000 Plovdiv, Bulgaria

${ }^{2}$ Faculty of Physics, Sofia University, 5, J.Bourchier blvd., Sofia-1164, Bulgaria

${ }^{3}$ Institute of General and Inorganic Chemistry, Bulgarian Academy of Sciences, Sofia-1113, Bulgaria

${ }^{4}$ Department of Physics, Imperial College London, London, UK

${ }^{5}$ Linköping University, Department of Physics, Chemistry \& Biology, 58183 Linköping, Sweden

*E-mail: vtd@phys.uni-sofia.bg

\begin{abstract}
We present a study of melt grown dilute nitride InGaAsN layers by X-ray photoelectron spectroscopy (XPS), Raman and photoluminescence (PL) spectroscopy. The purpose is to determine the degree of atomic ordering in the quaternary alloy during the epitaxial growth at near thermodynamic equilibrium conditions and its influence on the band gap formation. Despite the low In concentration $(\sim 3 \%)$ the XPS data show a strong preference of In-N bonding configuration in the InGaAsN samples. Raman spectra reveal that most of the $\mathrm{N}$ atoms are bonded to In instead of $\mathrm{Ga}$ atoms and the formation of $\mathrm{N}$-centred $\mathrm{In}_{3} \mathrm{Ga}_{1}$ clusters. PL measurements reveal smaller optical band gap bowing as compared to the theoretically predictions for random alloy and localized tail states near to the conduction band minimum.
\end{abstract}




\section{Introduction}

The great interest in InGaAsN alloys for more than two decades is due to their potential to alter the energy band gap and the lattice constant, which creates additional flexibility desired in many applications such as multijunction solar cells [1,2], heterojunction bipolar transistors [3] and high-speed photodetectors [4]. However, epitaxial growth of dilute nitride bulk layers remains a great challenge because of the low incorporation efficiency of $\mathrm{N}$ into the growing crystal, the large alloy miscibility gap in the phase diagram, the large difference in lattice constant between nitrides and arsenides and the local high strain near $\mathrm{N}$ atoms. These problems are, in part, responsible for the creation of nonradiative centres and structural inhomogeneity, resulting in general in poor luminescence efficiency of as-grown InGaAsN. Even at very small concentration the incorporation of nitrogen into crystal lattice deteriorates the crystalline quality of GaAs and dramatically changes the physical properties of the compounds $[5,6]$. The quality of the epitaxial layers depends strongly on the growth conditions. The epitaxial growth under extreme nonequilibrium conditions, such as molecular beam epitaxy (MBE) and metaloorganic chemical beam deposition (MOCVD), favours the formation of various defects responsible for many of the anomalous optical and transport properties of dilute nitride alloys [7-10]. In order to reduce defect densities and obtain more ordered alloy, additional annealing is performed [11-13].

Many theoretical methods have been applied to study the anomalous effects observed in III-V nitrides: The Band-Anticrossing model describes the electronic structure of dilute nitrides by considering the interaction between the delocalized states of the host semiconductor and the localized states of the highly electronegative nitrogen impurity, but ignores cluster states beyond the isolated nitrogen atoms [14]. The empirical pseudopotential theory describes the evolution of the electronic structure of GaAsN alloys, from the dilute impurity limit to the fully formed alloy [15]. Semi-empirical tight-binding model allows calculating the dependence of 
the optical and transport properties of the material on the nitrogen concentration in a wide energy range [16].

Up to now, few efforts have been performed to understand the local microstructure, i.e. possible atomic ordering in these alloys, which can dramatically alter their electronic and transport properties important for device applications. Kim and Zunger [17] have pointed out that the number of atomic bonds of each type in InGaAsN is not only related to the compositions as in the case of ternary alloys but also depends on the possible short-range atomic ordering in the quaternary compound. By means of Monte-Carlo simulations, these authors have analysed the different kinds of atomic bonds in InGaAsN and predicted a strong effect of the short-range ordering on the formation of the alloy band gap.

In this paper, we present an experimental study of the microstructure of InGaAsN layers grown from melt by liquid phase epitaxy (LPE) at near equilibrium conditions. For that purpose, we have investigated the structural and optical properties of as grown InGaAsN layers.

\section{Experimental details}

The InGaAsN samples under study were grown by low-temperature LPE on (100) n-type GaAs:Si $\left(\sim 10^{18} \mathrm{~cm}^{-3}\right)$ substrates. A series of samples was grown from mixed solution with composition of $90 \%$ In $+10 \% \mathrm{Ga}$, both with $6 \mathrm{~N}$ purity. Polycrystalline GaAs and GaN with purity of $5 \mathrm{~N}$ were used for sources of As and $\mathrm{N}$ respectively. The $\mathrm{N}$ content in the melt was 0.5 at. \%. The crystallization was carried out from initial epitaxy temperature in the range 590 $560{ }^{\circ} \mathrm{C}$ at a cooling rate of $1{ }^{\circ} \mathrm{C} / \mathrm{min}$ for $2-3$ minutes. The thickness of the layers measured on the cross-section by scanning electron microscopy (LYRA I XMU, Tescan) is in the range 2$2.5 \mu \mathrm{m}$. The In concentration measured by energy dispersive X-ray microanalyzer (Quantax, Bruker) is $\sim 3$ at. \%. High-resolution X-ray diffraction (XRD) method was used for determination of the lattice parameter, mismatch and crystalline quality of the layers. 
X-ray photoelectron spectroscopy (XPS) measurements were carried out on an AXIS Supra electron spectrometer (Kratos Analyical Ltd.) with base vacuum in the analysis chamber in the order of $10^{-8} \mathrm{~Pa}$. The samples were irradiated with monochromatized $\mathrm{Al} \mathrm{K}_{\alpha}$ photons with energy of $1486.6 \mathrm{eV}$. The photoemitted electrons were separated, according to their kinetic energy, by a $180^{\circ}$-hemispherical analyzer with a total instrumental resolution of $0.54 \mathrm{eV}$ (as measured by the FWHM of $\mathrm{Ag} 3 \mathrm{~d}_{5 / 2}$ line) at pass energy of $20 \mathrm{eV}$. Energy calibration was performed by normalizing the $\mathrm{C} 1 \mathrm{~s}$ line of adventitious adsorbed hydrocarbons to $285.0 \mathrm{eV}$. The diameter of the analysis area was $750 \mu \mathrm{m}$.

Photoluminescence (PL) measurements at $300 \mathrm{~K}$ were performed using laser excitation at $532 \mathrm{~nm}(2.33 \mathrm{eV})$ with $50 \mathrm{~W} / \mathrm{cm}^{2}$ power, a $0.5 \mathrm{~m}$ focal length spectrometer (Acton 2300i, Princeton Instruments) and a Si detector. PL at 2K and 109K was also measured using a Jobin Yvon monochromator (model HR460, f=460 mm) and $532 \mathrm{~nm}$ laser excitation. In this case, a much lower excitation power $\left(\sim 100 \mathrm{~mW} / \mathrm{cm}^{2}\right)$ was enough to obtain a strong enough PL signal, which was detected by a CCD camera.

Micro-Raman spectroscopy by means of a Jobin Yvone LabRAM HR800 spectrometer and laser excitation at $632 \mathrm{~nm}$ was applied in backscattering geometry to study the microscopic lattice structure related to the incorporation of $\mathrm{N}$ in InGaAsN alloys.

\section{Results and discussions}

\section{$\underline{\text { X-ray Diffraction }}$}

A typical XRD curve is plotted in figure 1. Two sharp peaks associated with the GaAs substrate and the InGaAsN layer are observed indicating high quality material. The lattice mismatch $\Delta a / a_{\mathrm{o}}$ determined from the XRD spectra is around $0.16 \%$. From the above depicted value, the concentration of In and the Vegard's rule the $N$-content was determined to be 0.3 at. $\%$. 


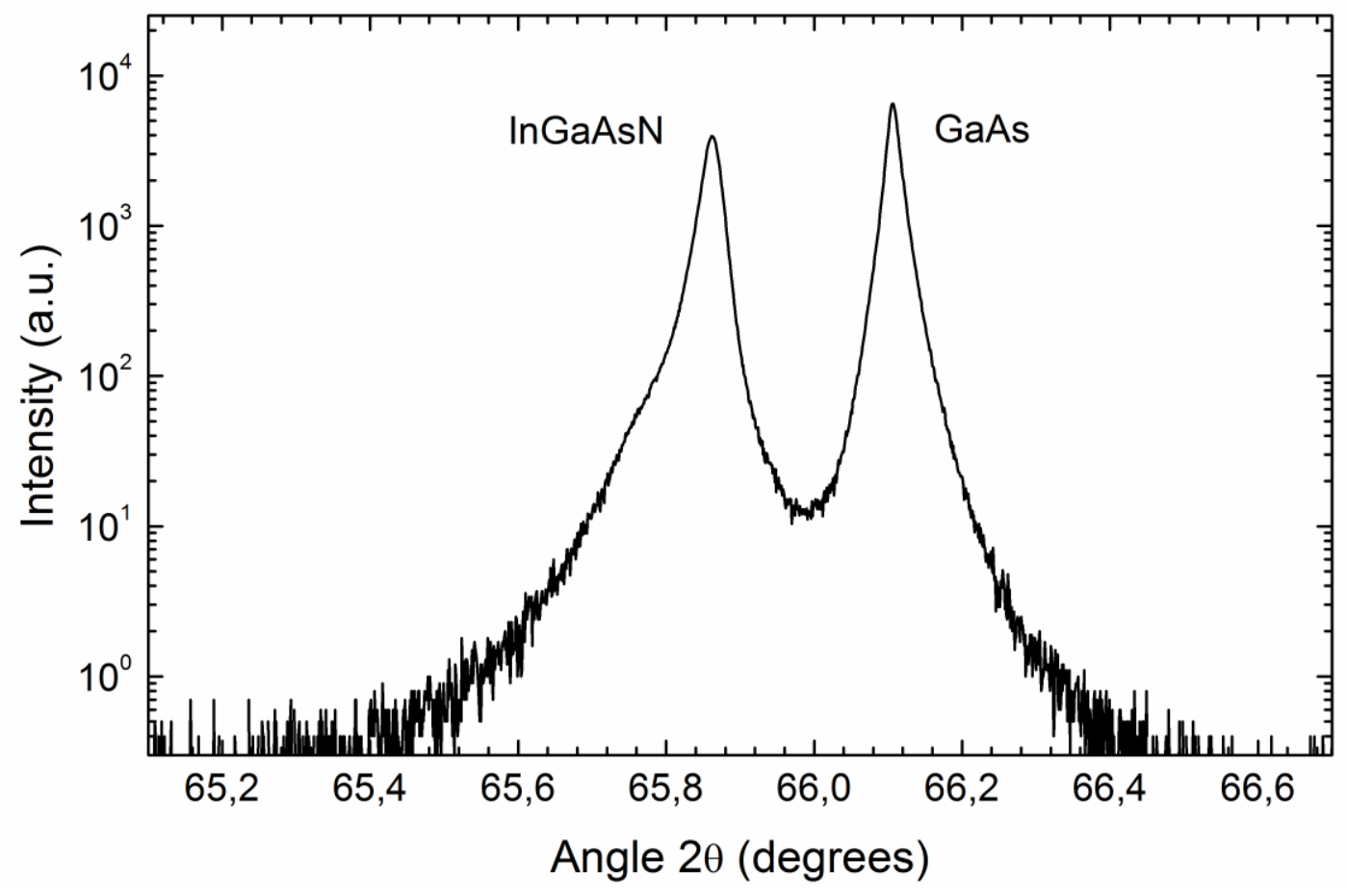

Figure 1. High-resolution X-ray diffraction (004) curve of an InGaAsN sample.

\section{$\underline{X-r a y ~ p h o t o e l e c t r o n ~ s p e c t r o s c o p y ~}$}

XPS is an efficient method to explore the nearest-neighbour bonding configuration for $\mathrm{N}$ in different compounds. However, the XPS analysis of the present samples is complicated because Ga Auger peaks appear in the same spectral region as the N $1 s$ photoelectron peak. In order to distinguish the N-related lines from the Ga Auger lines in the InGaNAs spectra a GaAs (001) reference sample was also analysed.

The surfaces of the InGaAsN layers and GaAs reference sample were cleaned in situ in the XPS chamber by $1 \mathrm{keV} \mathrm{Ar}^{+}$bombardment. The absence of atmospheric contaminations was established by monitoring the $\mathrm{C} 1 \mathrm{~s}, \mathrm{O} 1 s$, As $3 d$, As $2 p$, Ga $3 d$, and Ga $2 p$ photoelectron peaks. 


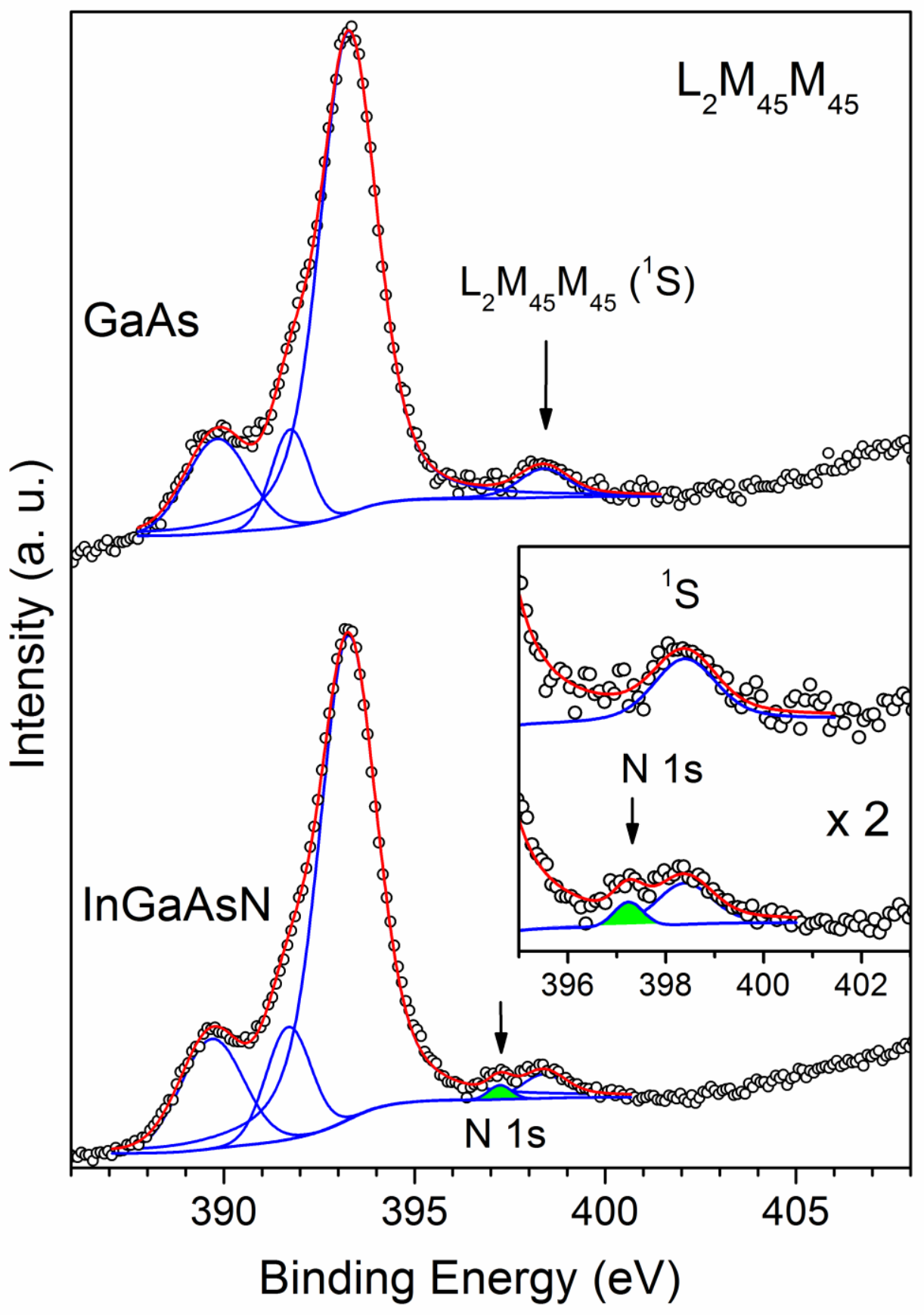

Figure 2. Ga LMM Auger region in the XPS spectra of the InGaAsN and GaAs samples. The inset shows closer views of the region around the N1s peak, revealing N-In bonding configuration. The different peak contributions are coloured in blue and their sum in red. The $\mathrm{N}$ 1s-peak area (in the low spectrum) is filled in green. 
In figure 2 the photoelectron spectra of InGaAsN and referent GaAs samples are compared in the binding-energy range from 386 to $408 \mathrm{eV}$. The dominant features are the $\mathrm{Ga}$ $\mathrm{L}_{2} \mathrm{M}_{45} \mathrm{M}_{45}$ Auger peaks, which overlap the $\mathrm{N}$ 1s contribution. In order to detect reliably the $\mathrm{N}$ 1s peak characteristic for InGaAsN, the complex Ga LMM structures of both samples are fitted with their different transition-term contributions according to the detailed analysis of Antonides et al. [18]. Intense Ga LMM contributions centred at 389.7, $393.3 \mathrm{eV}$ and at $398.4 \mathrm{eV}$ are seen in the spectra. The comparison with the GaAs referent sample allows detecting the N1s corelevel peak at $397.2 \mathrm{eV}$ in the spectrum of the $\mathrm{InGaAsN}$ sample. This peak is partially overlapped with the contribution of the ${ }^{1} \mathrm{~S}$ final-state term in the $\mathrm{L}_{2} \mathrm{M}_{45} \mathrm{M}_{45}$ Auger process.

In the ideal case $\mathrm{N}$ has five different nearest-neighbour configurations for InGaAsN, and their binding energies are closely distributed in an interval of $1 \mathrm{eV}$ from $397.0 \mathrm{eV}$ (energy of the 4-In configuration) to $398.0 \mathrm{eV}$ (energy of the 4-Ga configuration) [19,20]. The observation of the $397.2 \mathrm{eV}$ peak in figure 3 indicates that $\mathrm{N}-\mathrm{In}$ is the dominant bonding configuration in our InGaAsN samples. This finding is in good agreement with the theoretical analyses of the microscopic lattice structures related to the incorporation of $\mathrm{N}$ in $\mathrm{InGaAsN}$ alloys performed by Kim and Zunger by means of Monte-Carlo simulations [17]. These authors have found that the equilibrium configuration of the alloy that minimizes the sum of strain plus "chemical" (bond) energies is $(\mathrm{Ga}-\mathrm{As})+(\mathrm{N}-\mathrm{In})$ bond configuration, i.e. it is energetically more favourable to form ordered alloy GaAs $+\mathrm{InN}$. This type of short range ordering results in statistically enhanced concentration of $\mathrm{N}$-centred $\mathrm{In}_{3} \mathrm{Ga}_{1}$ clusters.

The samples under study are grown by LPE, which is carried out under near to equilibrium conditions. For that reason, having in mind the theoretical predictions from [17] we assume that the Ga-As configuration is preferred for better lattice matching of the alloy to the GaAs substrate and N-In bonds are more favourable since they reduce the local strain. The short range ordering and the dominance of $\mathrm{N}$-centred $\mathrm{In}_{3} \mathrm{Ga}_{1}$ clusters is an important feature of 
quaternary InGaAsN isovalent systems, which influences the optical properties of the alloys [17]. This is evidenced below in the discussion of the results from Raman and PL measurements.

\section{$\underline{\text { Raman spectroscopy }}$}

Raman spectroscopy is a direct method for studying the local environment of nitrogen atoms in dilute nitrides. Figure 3 displays the Raman spectrum of an InGaAsN sample. In addition to the GaAs-like LO and TO phonons, the spectrum reveals acoustic modes as well as second-order signatures due to the relaxation of the Raman selection rules caused by the nitrogen-induced disorder. One does not observe $\mathrm{N}$-induced local vibration modes (LVM) around $472 \mathrm{~cm}^{-1}$, associated with isolated nitrogen atom bonded to four Ga neighbours $\left(\mathrm{N}_{\mathrm{As}} \mathrm{Ga}_{4}\right)$. Instead, a LVM peak at $447 \mathrm{~cm}^{-1}$ originating from $\mathrm{N}$-In bonds appears. A similar LVM peak has been reported to occur as a new additional feature for MBE grown InGaAsN after annealing at high temperatures [21]. This frequency is close to the value of $443 \mathrm{~cm}^{-1}$ of the nitrogen mode in InAs. It has been found theoretically and experimentally that in as-grown Inrich low Ga-content dilute InGaAsN alloys a complete change from pure In-N bonding $\left(\mathrm{N}_{\mathrm{As}} \mathrm{In}_{4}\right)$ to the preferential bonding in $\mathrm{N}$-centred $\operatorname{In}_{3} \mathrm{Ga}_{1}$ clusters occurs $[22,23]$. On the other hand for Ga-rich InGaAsN samples grown by MBE and MOCVD additional LVM bands between 472 and $443 \mathrm{~cm}^{-1}$ have been observed after annealing [24-27]. Since the recorded LVM peak of our samples is close to those of In-rich compounds we can assume that the N-centred $\operatorname{In}_{3} \mathrm{Ga}_{1}$ clusters are the dominant configuration for $\mathrm{N}$ atoms, which is in accordance with the XPS results. Obviously, the LPE growth at near equilibrium condition tends to form an indium-rich environment for the nitrogen atom even for low In content compounds. 


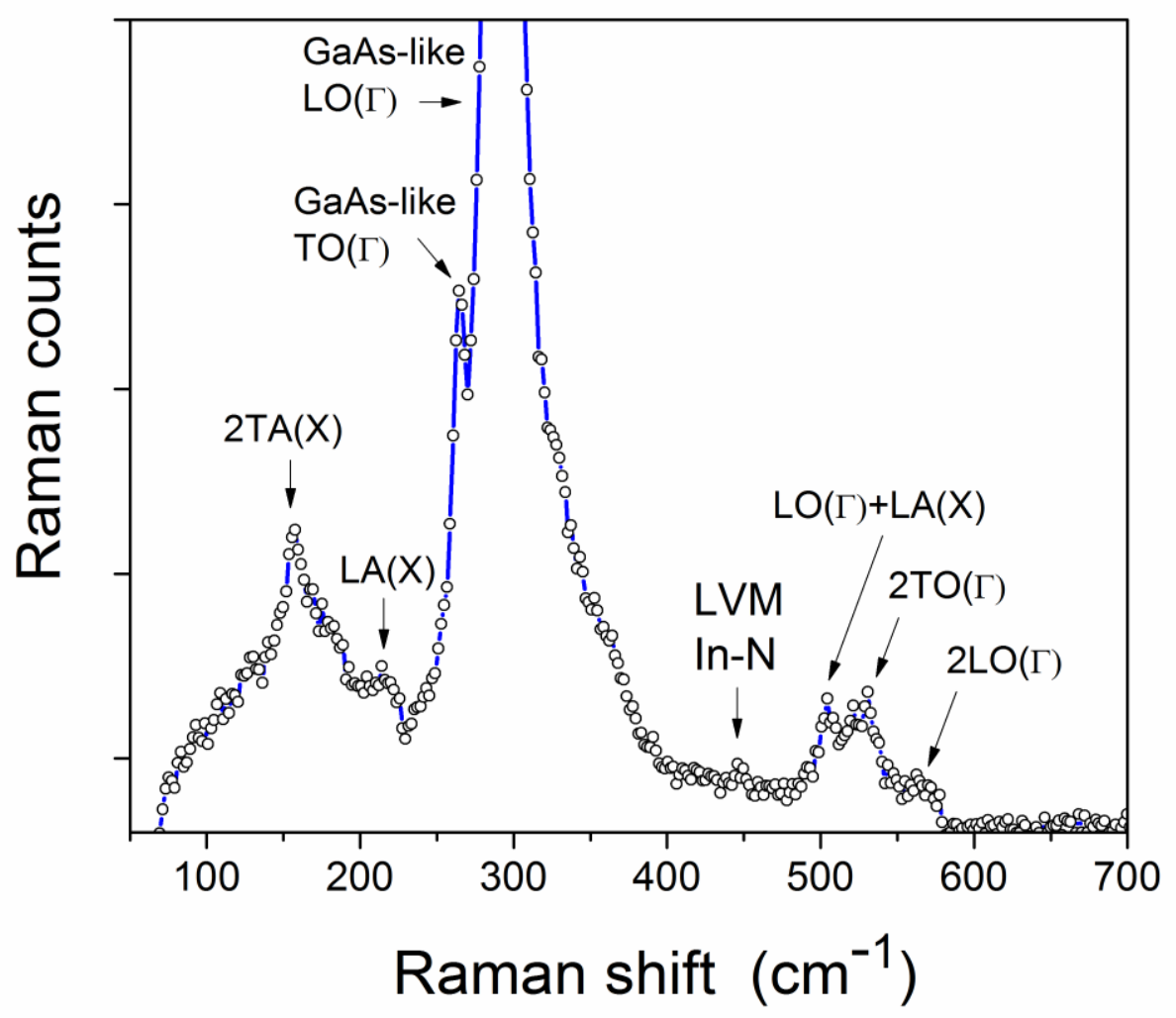

Figure 3. Raman spectrum of an as grown InGaAsN layer.

\section{Photoluminescence spectroscopy}

Typical PL spectra of the InGaAsN samples measured at $2 \mathrm{~K}, 109 \mathrm{~K}$ and $300 \mathrm{~K}$ are presented in figure 4 . The PL spectral shape at $2 \mathrm{~K}$ consists of a sharp peak at $1.459 \mathrm{eV}$ with a low-energy tail. We suggest that two carrier recombination mechanisms are responsible for the PL emission. The sharp high-energy peak is due to the near band-edge luminescence, while the low-energy tail originates from the recombination of photogenerated carriers trapped by defect states in the conduction band tail of the InGaNAs layer. N-related defect states, both localized and extended, present very close to the band edge, and their mixing with the band-edge provide an asymmetry to the luminescence spectrum on the low-energy side as discussed in [28]. 


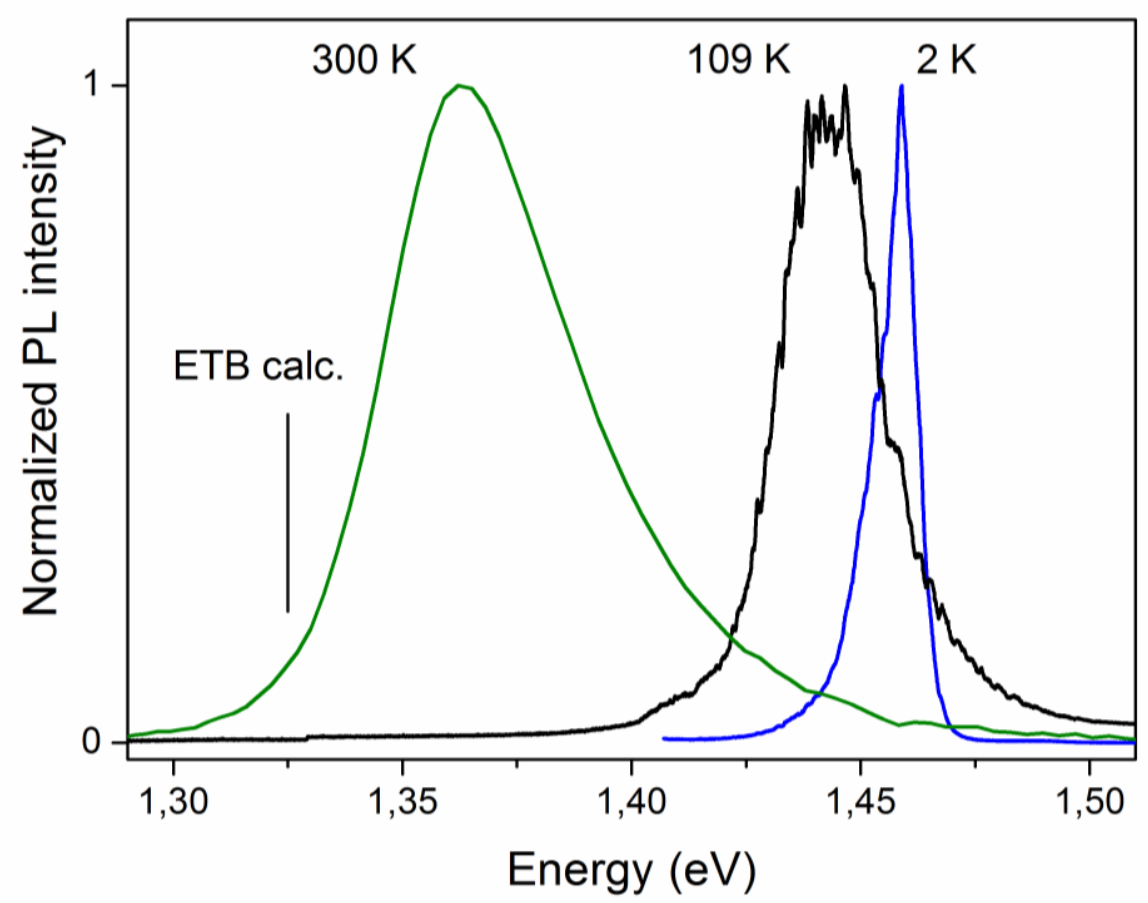

Figure 4. Normalized PL spectra of an InGaAsN sample measured at $2 \mathrm{~K}, 109 \mathrm{~K}$ and $300 \mathrm{~K}$.

At temperature of $109 \mathrm{~K}$ the PL peak shifts to lower energies and decreases in magnitude by a factor of 45 (not shown here). It is broader and reveals many small peaks and shoulders on its both sides. The thermal quenching of PL is attributed to the activation of nonradiative channels associated with $\mathrm{N}$-related defects [29]. In addition, the temperature facilitates the trapping in deeper recombination centres and this, (together with the bandgap decreasing), accounts for the red shift and broadening of the PL peak. Further increase of the temperature to $300 \mathrm{~K}$ leads to a much broader emission peak centred at $1.362 \mathrm{eV}$. The red shift with respect to $2 \mathrm{~K}$ is due to band gap narrowing with increasing temperature. An exponential tail is present at the high-energy side of the peak resulting from the Boltzmann carrier distribution.

The bandgap values determined from the PL peak positions are larger than the value $(1.325 \mathrm{eV})$ calculated for random dilute nitride alloy (with the same composition) by the empirical tight binding method with the use of virtual crystal approximation for the tight binding parameters of the InGaAs ternary [30]. The calculated value is indicated by a vertical 
line in figure 4. This discrepancy could be explained taking into account the above mentioned theoretical analysis of Kim and Zunger [17], which has shown that the band gap formation of the quaternary InGaAsN depends not only from the composition, but also from the short range ordering of the atoms. The main results of this local ordering are an increase of the band gap with respect to the random alloy case and the appearance of a tail of localized states around the conduction band minimum due to different clusters of nitrogen atoms surrounded by varying number of indium and gallium atoms. As stated above, LPE growth at nearly equilibrium conditions favours this short range ordering, which main effect on the band gap formation of the grown layers is reducing the optical bowing as compared to random alloys.

\section{Conclusion}

Dilute nitride InGaAsN layers with low In concentration ( 3 at. \%) and thicknesses in the range $2-2.5 \mu \mathrm{m}$ have been grown on GaAs substrates by low-temperature LPE. The nitrogen content in the layers determined from XRD diffraction curves is about 0.3 at. \%. The nitrogen bonding configurations have been studied by means of XPS and Raman spectroscopy. The N(1s) core-level photoelectron spectra show a peak, corresponding to binding energy of $397.2 \mathrm{eV}$ and associated with preferential In-N bonds, which indicates that $\mathrm{N}$ has a bonding configuration with In-rich nearest neighbours in our InGaAsN samples. This is in agreement with the Raman spectra that exhibit a LVM band around $447 \mathrm{~cm}^{-1}$ due to the vibration of nitrogen in $\mathrm{N}$-centred $\mathrm{In}_{3} \mathrm{Ga}_{1}$ clusters. The effect of this micro-cluster local ordering is the increasing of the bandgap with respect to the random alloy case, as revealed by PL data.

The present results are in a good agreement with the major conclusions of the theoretical analysis from [17] about the existence of local atomic ordering in quaternary InGaAsN alloys occurring at equilibrium conditions and its influence on their optical properties. It contributes 
to a better interpretation of the experimental results obtained in LPE grown dilute nitrides and better understanding of their properties.

\section{Acknowledgements}

This work was supported by the Cost Action MP1406 "Multiscale in modelling and validation for solar photovoltaics (MultiscaleSolar)" and the Bulgarian National Science Fund (contract ДКОСТ 01/16). The authors acknowledge A. Tsonev and K. Genkov (Sofia University) for the EDX and SEM measurements and J.W. Gerlach (Leibniz Institute for Surface Modification) for the HRXRD measurements.

\section{References}

[1] Kurtz S R, Allerman A A, Jones E D, Gee J M, Banas J J and Hammons B E 1999 InGaAsN solar cells with $1.0 \mathrm{eV}$ band gap, lattice matched to GaAs Appl. Phys. Lett. 74 729-31

[2] Aho A, Polojärvi V, Korpijärvi V-M, Salmi J, Tukiainen A, Laukkanen P and Guina M 2014 Composition dependent growth dynamics in molecular beam epitaxy of GaInNAs solar cells Sol. Energy Mater. Sol. Cells 124 150-8

[3] Asbeck P M, Welty R J, Tu C W, Xin H P and Welser R E 2002 Heterojunction bipolar transistors implemented with GaInNAs materials Semicond. Sci. Technol. 17 898-906

[4] Chen W C, Su Y K, Chuang R W, Yu H C, Chen B Y and Hsu S H 2008 Investigation of InGaAsN MSM photodetectors with transparent ITO Schottky contacts Semicond. Sci. Technol. 2335027

[5] Zhang, S B, Wei S-H 2004 Theory of Defects in dilute nitrides Physics and Applications of Dilute Nitrides ed Irina A Buyanova and Weimin M Chen (New York) 
pp 239-70

[6] Krispin P, Gambin V, Harris J S and Ploog K H 2003 Nitrogen-related electron traps in $\mathrm{Ga}(\mathrm{As}, \mathrm{N})$ layers $(<<3 \% \mathrm{~N})$ J. Appl. Phys. 93 6095-9

[7] Kwon D, Kaplar R J, Ringel S A, Allerman A A, Kurtz S R and Jones E D 1999 Deep levels in p-type InGaAsN lattice matched to GaAs Appl. Phys. Lett. 74 2830-2832

[8] Chen W M, Buyanova I A, Tu C W and Yonezu H 2006 Point defects in dilute nitride III-N-As and III-N-P Phys. B Condens. Matter 376-377 545-51

[9] Thinh N Q, Buyanova I A, Chen W M, Xin H P and Tu C W 2001 Formation of nonradiative defects in molecular beam epitaxial GaNxAs1-x studied by optically detected magnetic resonance Appl. Phys. Lett. 79 3089-91

[10] Xie S Y, Yoon S F and Wang S Z 2005 Photoluminescence properties of p-type InGaAsN grown by RF plasma-assisted molecular beam epitaxy Appl. Phys. A 81 98790

[11] Tan S L, Hunter C J, Zhang S, Tan L J J, Goh Y L, Ng J S, Marko I P, Sweeney S J, Adams A R, Allam J and David J P R 2012 Improved Optoelectronic Properties of Rapid Thermally Annealed Dilute Nitride GaInNAs Photodetectors J. Electron. Mater. $413393-401$

[12] Li W, Pessa M, Ahlgren T and Decker J 2001 Origin of improved luminescence efficiency after annealing of $\mathrm{Ga}(\mathrm{In}) \mathrm{NAs}$ materials grown by molecular-beam epitaxy Appl. Phys. Lett. 79 1094-6

[13] Biswas M, Shinde N, Makkar R L, Bhatnagar A and Chakrabarti S 2017 Varying nitrogen background pressure; an efficient approach to improve electrical properties of MBE-grown GaAs1-xNx thin films with less atomic disorder J. Alloys Compd. 695 $3163-9$

[14] Shan W, Walukiewicz W, Ager J W, Haller E E, Geisz J F, Friedman D J, Olson J M 
and Kurtz S R 1999 Band Anticrossing in GaInNAs Alloys Phys. Rev. Lett. 82 1221-4

[15] Kent P R C, Bellaiche L and Zunger A 2002 Pseudopotential theory of dilute III V nitrides Semicond. Sci. Technol. 17 851-9

[16] Shtinkov N, Desjardins P and Masut R 2003 Empirical tight-binding model for the electronic structure of dilute GaNAs alloys Phys. Rev. B 6781202

[17] Kim K and Zunger A 2001 Spatial correlations in GaInAsN alloys and their effects on band-gap enhancement and electron localization Phys. Rev. Lett. 86 2609-12

[18] Antonides E, Janse E C and Sawatzky G A 1977 LMM Auger spectra of Cu, Zn, Ga, and Ge. I. Transition probabilities, term splittings, and effective Coulomb interaction Phys. Rev. B 15 1669-79

[19] Lay T S, Kuo W T, Chen L P, Lai Y H, Hung W H, Wang J S, Chi J Y, Shih D K and Lin H H 2004 Probing the electronic structures of III-V-nitride semiconductors by Xray photoelectron spectroscopy J. Vac. Sci. Technol. B Microelectron. Nanom. Struct. 221491

[20] Veal T D, Mahboob I, Piper L F J, McConville C F and Hopkinson M 2004 Core-level photoemission spectroscopy of nitrogen bonding in GaNxAs1-x alloys Appl. Phys. Lett. 85 1550-2

[21] Alt H C and Gomeniuk Y V. 2004 Local mode frequencies of the NAs-InGa nearestneighbor pair in (Ga,In)(As,N) alloys [19] Phys. Rev. B $701-4$

[22] Talwar D N 2008 Assessing the Preferential Chemical Bonding of Nitrogen in Novel Dilute III-As-N Alloys Dilute III-V Nitride Semiconductors and Material Systems ed A Erol. (Springer-Verlag Berlin Heidelberg) pp 223-53

[23] Wagner J, Köhler K, Ganser P and Maier M 2005 Bonding of nitrogen in dilute InAsN and high In-content GaInAsN Appl. Phys. Lett. 8751913

[24] Mintairov A M, Blagnov P A, Merz J L, Ustinov V M and Vlasov A S 2001 
Vibrational study of nitrogen incorporation in InGaAsN alloys Proceedings 9th Int. Symp. "Nanostructures: Physics and Technology” NC.13p, St Petersburg, Russia pp $238-41$

[25] Hashimoto A, Kitano T, Nguyen A K, Masuda A, Yamamoto A, Tanaka S, Takahashi M, Moto A, Tanabe T and Takagishi S 2003 Raman characterization of lattice-matched GaInAsN layers grown on GaAs (001) substrates Sol. Energy Mater. Sol. Cells 75 3137

[26] Kurtz S, Webb J, Gedvilas L, Friedman D, Geisz J, Olson J, King R, Joslin D and Karam N 2001 Structural changes during annealing of GaInAsN Appl. Phys. Lett. 78 $748-50$

[27] Pavelescu E-M, Wagner J, Komsa H-P, Rantala T T, Dumitrescu M and Pessa M 2005 Nitrogen incorporation into GaInNAs lattice-matched to GaAs: The effects of growth temperature and thermal annealing J. Appl. Phys. 9883524

[28] Bhuyan S, Das S K, Dhar S, Pal B and Bansal B 2014 Optical density of states in ultradilute GaAsN alloy: Coexistence of free excitons and impurity band of localized and delocalized states J. Appl. Phys. 116

[29] Kudrawiec R, Sk G, Misiewicz J, Ishikawa F, Trampert A and Ploog K H 2009 Localized and delocalized states in GaNAs studied by microphotoluminescence and photoreflectance Appl. Phys. Lett. $942-4$

[30] Donchev V, Asenova I, Milanova M, Alvarez D A, Kirilov K, Shtinkov N, Ivanov I G, Georgiev S, Valcheva E and Ekins-Daukes N 2017 Optical properties of thick GaInAs (Sb) N layers grown by liquid-phase epitaxy J. Phys. Conf. Ser. 79412013 